\title{
RAINFALL ENHANCEMENT THROUGH CLOUD SEEDING: A CASE STUDY
}

\author{
MANIKIAM BALAKRISHNAN ${ }^{1}$, V. SHIVA PRAKASH ${ }^{2}$, \\ PRAKASH KUMAR ${ }^{3} \&$ CHIDANANDA MURTHY ${ }^{3}$ \\ ${ }^{1}$ Bangalore University, India \\ ${ }^{2}$ Karnataka State Disaster Management Centre, India \\ ${ }^{3}$ Rural Water Supply \& Sanitation Board, Karnataka, India
}

\begin{abstract}
Karnataka state in India is highly prone to drought conditions due to the high variability of rainfall. The main rainfall season is during the monsoon period of June to September. With most of the agriculture being rain-fed, the impact of deficient rainfall is very high. Most of Karnataka is in the rain shadow region due to being in the leeward side of the Western Ghats mountain range. This leads to large amounts of clouding over Karnataka with potential or sub critical potential for rainfall. The monsoon rainfall in 2017 was deficient for the entire state until mid-August and this provided a suitable occasion for taking up cloud seeding operations. Towards mitigating this adverse impact, the Government undertook a project on cloud seeding using modern techniques. The infrastructure for the project consisted of installation of the Doppler radars at three strategic locations: Bengaluru, Gadag and Shorapur to monitor clouds over the entire state. The observations were supported by Radiosonde equipment at Gadag, Bengaluru and Sholapur (closer to Shorapur). The hourly INSAT satellite data provided cloud cover, cloud top temperature, cloud motion vector and potential water content. The model forecasts for the day issued by the Indian Meteorological Department and the Indian Space Research Organisation using high resolution model runs were also utilized. All these data were analysed to prepare a potential area chart indicating areas for cloud seeding based on antecedent rainfall, cloud convection, synoptic situation and current radar observations. The final selection of the cloud system was based on aircraft observations of temperature, liquid water content and updrafts. The rainfall monitoring over the state was carried out using a calibrated dense network of automated telemetric rainguage network of 6,000 stations. The results from several case studies are presented in this paper. The quantitative results indicate positive impact of cloud seeding and a viable alternative to recurrent drought hit areas.
\end{abstract}

Keywords: cloud, seeding, convection, satellite, drought, rainfall, rainguage, radar, weather model, evaluation.

\section{INTRODUCTION}

Over the past two decades, technology innovations have led to developing techniques to seed clouds using aircrafts and induce rainfall. The cloud studies over the years have led to better understanding of the processes related to precipitation especially the role of aerosols in condensation of water vapour leading to precipitation. The cloud seeding experiments carried out ([1]-[5]) have assisted in developing a protocol for identifying potential target clouds using satellite and radar observations ([5], [6]) and using aircrafts, spray seeds in the form of sodium chloride nano particles at the base of the developing clouds which eventually leads to enhanced precipitation. The cloud seeding operation was taken up in the state of Karnataka in India in 2017 to enhance monsoon rainfall.

The state of Karnataka in India consists of regions of Malnad and coastal Karnataka that receive copious amount of rainfall; its north region in the Deccan Plateau which is most arid regions in the country. Most of the rains received in the state is during the monsoon season. Being an agrarian economy with a large percentage of its citizens engaged in agriculture, the failure of rains can have a crippling effect on the economy of the state. 
Monsoon rainfall begins in June and continues until September. The annual rainfall over Karnataka has high variability as can be seen from the district wise distribution (Fig. 1).

During the 2017 season, the monsoon rainfall was very much below the normal for the months of June and July and it was decided to take up cloud seeding to enhance the rainfall specially to meet the agricultural needs.

\section{PROJECT AREAS AND FACILITIES}

The whole state of Karnataka was taken up for cloud seeding operations and the Weather Modification, LLC team from USA carried out the cloud seeding operations using two aircrafts namely B-200-N267CB and C-90-N6111V both Beachcrafts with appropriate seeding equipment. Three weather radars were set up at Bengaluru, Gadag and Shorapur to cover the entire Karnataka. The radars operated by scanning the target area every 7 minutes and giving cloud data, radar reflectivity, water content, convective index, etc. GPS Sonde flights were operated at these stations to study the atmospheric conditions and stability index. A team of meteorologists provided daily forecast of weather conditions and identified priority sites for cloud seeding by using high resolution model forecasts, satellite observations from INSAT 3D and radar images.

The areas of priority for seeding was decided on a day to day basis taking into account the synoptic weather situation, the cloud development especially convection using the GPS Sonde data, the real time cloud cover and characteristics using the half hourly satellite images from INSAT 3D satellite, zeroing in on the actual target cloud for seeding through radar data from three locations Bengaluru, Gadag and Shorapur. Three C-band radars are operated to cover the maximum area of the state. The radars are calibrated under the supervision of the radar experts. The radar reflectivity data was uploaded on the website and was made available on real time. The composite picture of reflectivities of the three radars is prepared to provide spatial and temporal distribution of seedable clouds in the state. The target cloud mass is studied through radar cross section data to evaluate potential liquid water content and phase of development. The outputs from high resolution $(3 \mathrm{~km}) \mathrm{WRF}$ model for short term prediction of cloud microphysical parameters were made use of for precise targeting of potential seedable clouds. This information on real time was given to pilots for seeding at short notice.

The INSAT hourly data is used in cloud seeding operations to identify potential areas as shown in Fig. 2.

Based on the weather data and model outputs, the priority target areas for cloud seeding are identified and maps are prepared with priority levels. These maps (Fig. 3) are used by pilots for flight planning for the day and seeding operations.

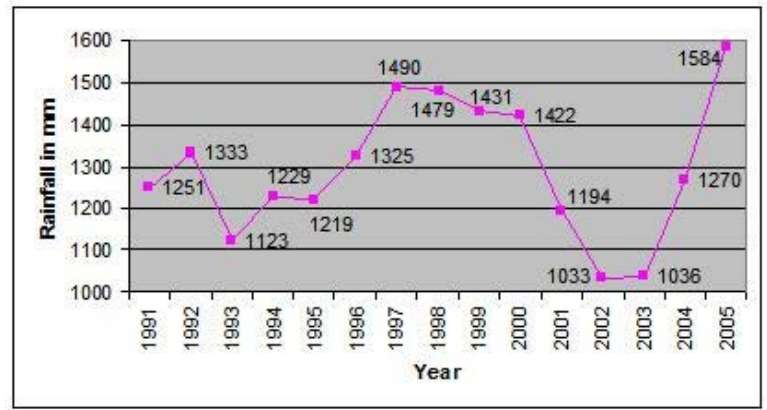

Figure 1: Rainfall variability over Karnataka state. 


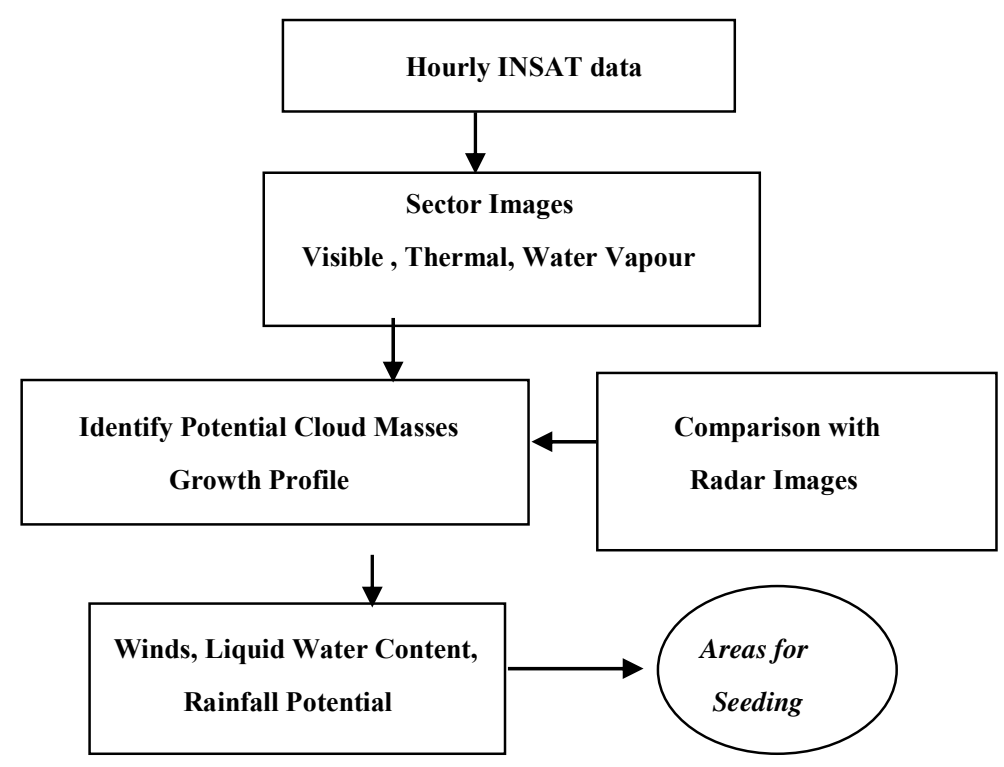

Figure 2: Schematic of identification of target cloud for seeding.

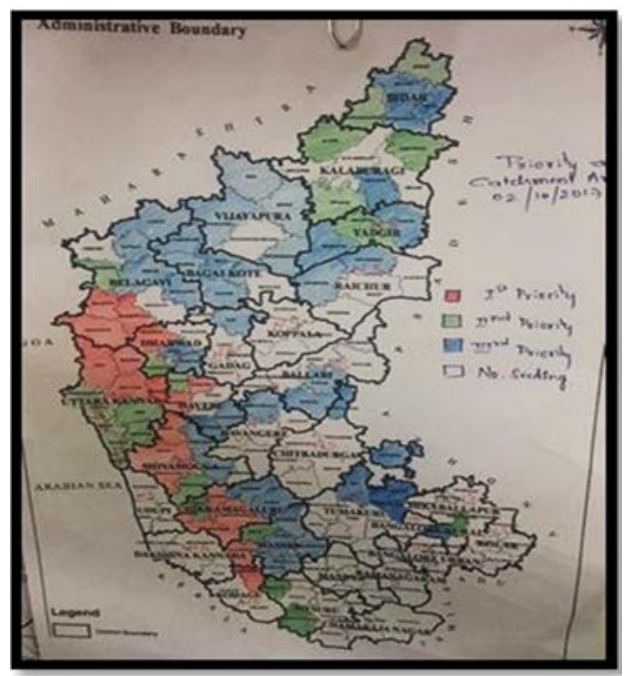

Figure 3: Karnataka priority seeding map and radar coverage (4th October 2017).

\section{CASE STUDIES}

A few case studies are given below to highlight the operations of cloud seeding. On 2nd October 2017 the forecast was issued by meteorologist as per operations protocol incorporating the project sounding data provided as well as the WMI modeling product which provides detailed cloud seeding specific parameters in order to determine convective areas, temperature levels and activity location for Karnataka. RAIN 1 aircraft was dispatched 
following the tracking and anticipation of seeding opportunity northwest of Hubli (Fig. 4). The following sequence of TITAN radar images and associated visual pictures provide an oversight of successful cloud top seeding in Karnataka.

During this seeding mission, the pilots were reporting $1000 \mathrm{fpm}$ (foot per minute) updrafts with ample supply of super-cooled liquid water to the point of getting ice accumulation on the aircraft during cloud penetrations. These physical observations are positive indications of glaciogenic seeding conditions which can be quite successful to which the correlated radar images also support. Radar images of the same mission were used by pilots of RAIN 1 to demonstrate the physical characteristics of successful seeding clouds as seen in Fig. 5.

Once aircraft is on location, experienced pilots fly into specific clouds to find vertical updrafts and super-cooled liquid water. This is a favourable location for seeding which transfers the atmospheric water into precipitation.

The cloudy regions which were selected for seeding operations may be seen as blue rings in the radar images (Fig. 6) and the development of the clouds after seeding leading to precipitation.

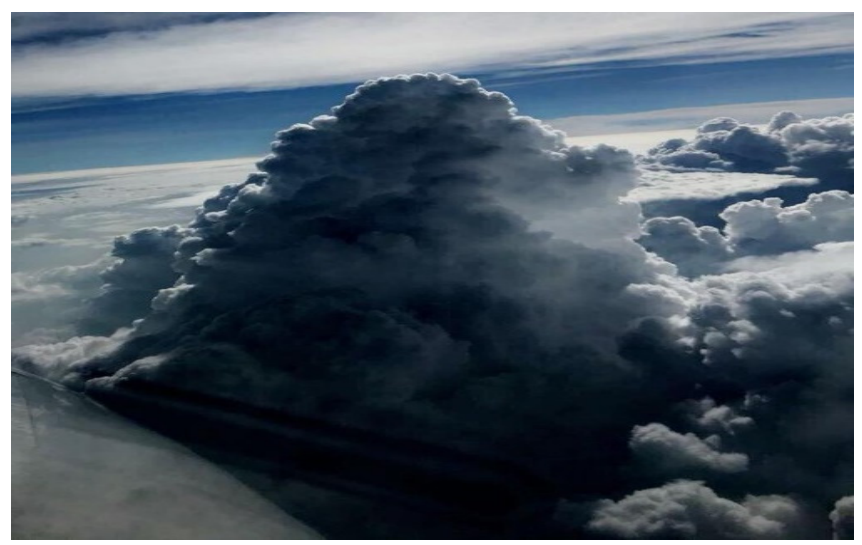

Figure 4: Convective cloud development over Hubbali, Karnataka.
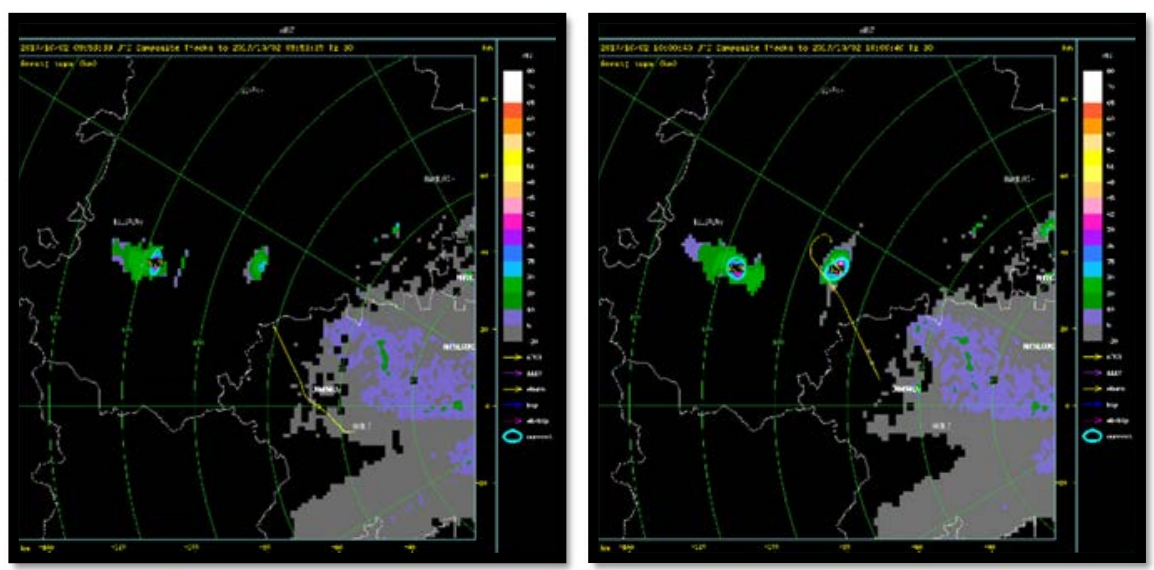

Figure 5: Convective clouds as seen in Radar images being seeded. 

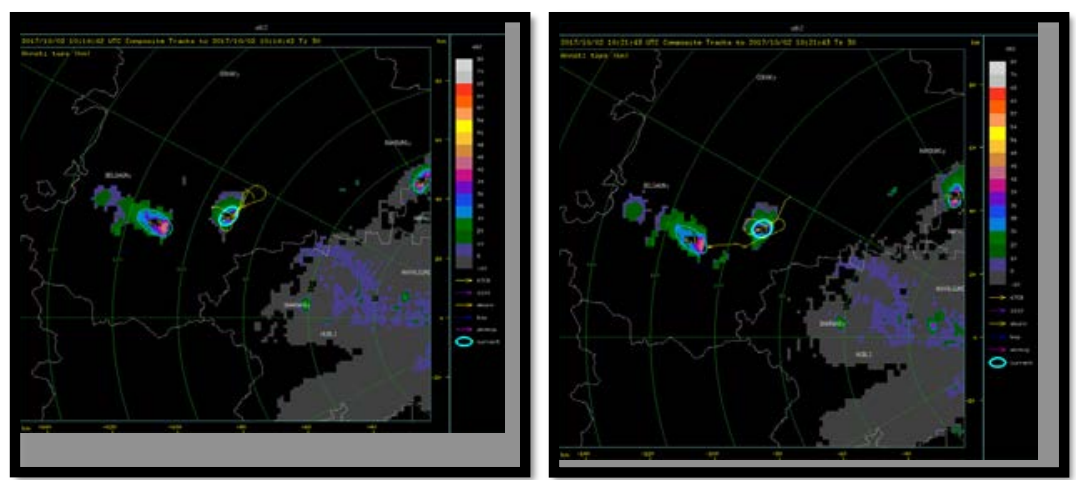

Figure 6: Convective target clouds for cloud seeding.
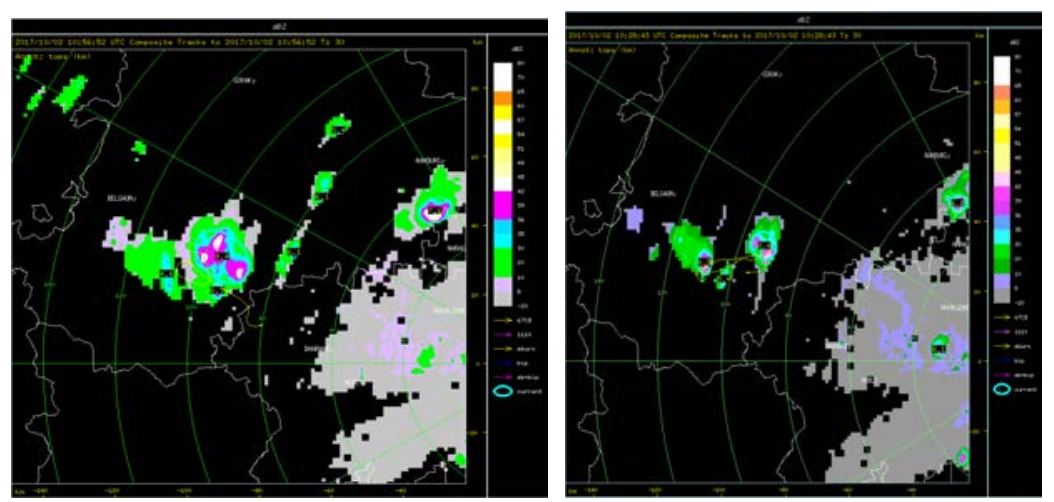

Figure 7: Pilots report positive seeding conditions and light flares to disperse cloud seeding materials. Radar images showing growing reflectivity and associated precipitation footprint.

The rainfall data from the high-resolution network of rainguages was collected for a period of one hour around the seeding time (Figs 6 and 7) and for a region of $50 \mathrm{~km}$ around the seeding location and analysed to study the impact of cloud seeding.

\section{EVALUATION OF CLOUD SEEDING}

\subsection{Preliminary analysis}

Cloud seeding programs have been conducted in India by different states for last many years using aerial seeding. The program was also conducted in the Karnataka state in the monsoon season of 2003. Rainfall data from 6,000 automatic rain gauges at high spatial and temporal scale is available for studying impact of seeding. The rainfall data will be also useful for research on rainfall estimation based on radar reflectivity. Daily advice on the priority areas for seeding operations based on accumulated rainfall at Hobli level and 2-meter-deep soil moisture. Evaluation of the seeding program is carried out by two ways - physical and statistical. 
Radar data is used for the physical evaluation of the seeding effects. The studies have shown that changes occur in the cloud after seeding. These are: increase in volume, increase in life time of cloud, increase in the intensity of the reflectivity, increase in the rain etc. These parameters are compared with the similar parameters of non-seeded clouds. It is observed that all the clouds in the area are not possible to seed. Because of aircraft flying restrictions, ATC permissions, not allowed to fly over restricted areas, etc., some seedable clouds are not targeted. These clouds are taken as control clouds. The parameters of seeded clouds (target clouds) are compared with the unseeded clouds (control clouds) to estimate the impact of seeding. The comparison is done with similar clouds. Comparison on daily basis guarantees the same large-scale background environment and removes bias due to due to day to day variability in the weather conditions.

In this method, rainfall data from target and control clouds is used to develop regression equation relationship between rainfalls from the target clouds and control clouds. This regression equation is applied to the seeded period to estimate to what the rainfall from seeded cloud would have been without seeding. This rainfall is considered as the rainfall due to natural process. The difference between actual rainfall and estimated rainfall gives the quantification of the seeding produced rainfall.

\subsection{Salient results}

Figs 8 and 9 shows the growth of seeded cloud with hygroscopic material, after seeding on 17th September 2017 seeding event. Fig. 9 shows growth in cloud parameters viz. cloud reflectance and precipitation. These figures indicate that clouds respond positively to the hygroscopic seeding.

The radar images show the variation in cloud reflectance and consequential rainfall that occurred in ground during a typical cloud seeding operations.
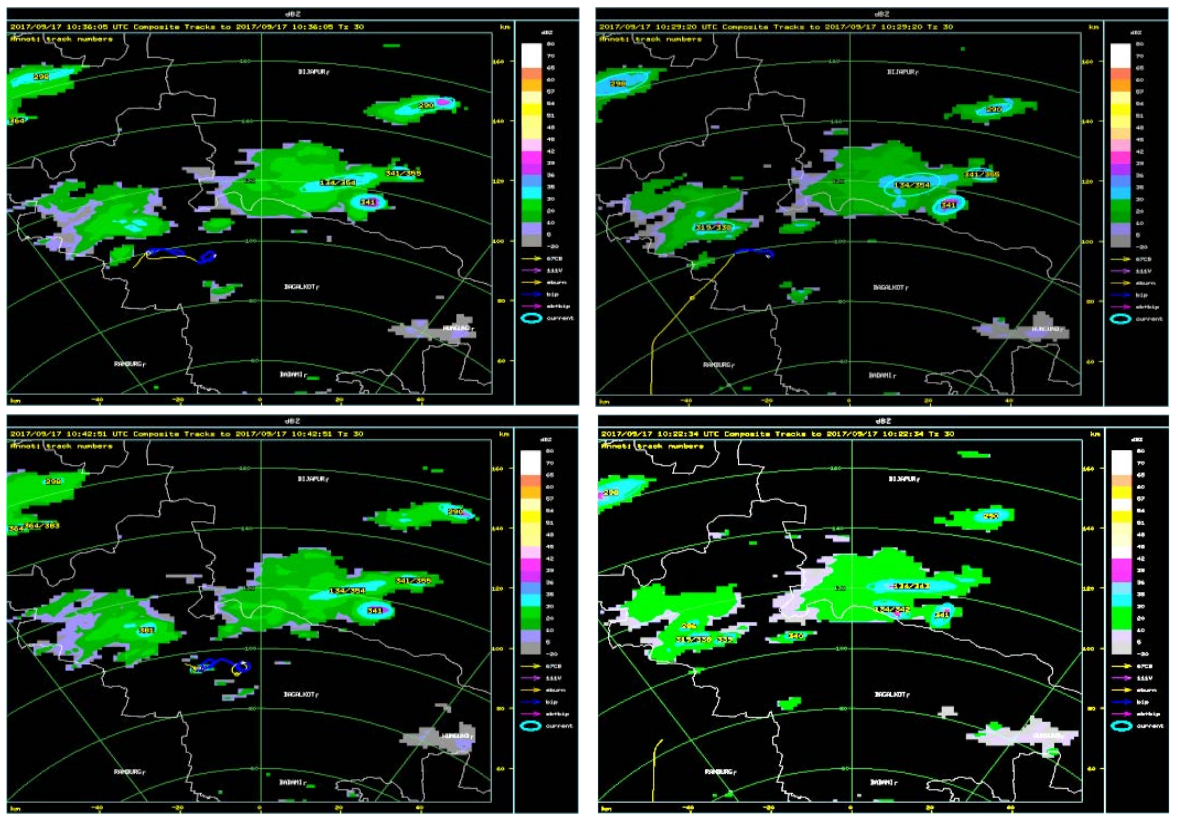

Figure 8: Sequential radar images during cloud seeding. 


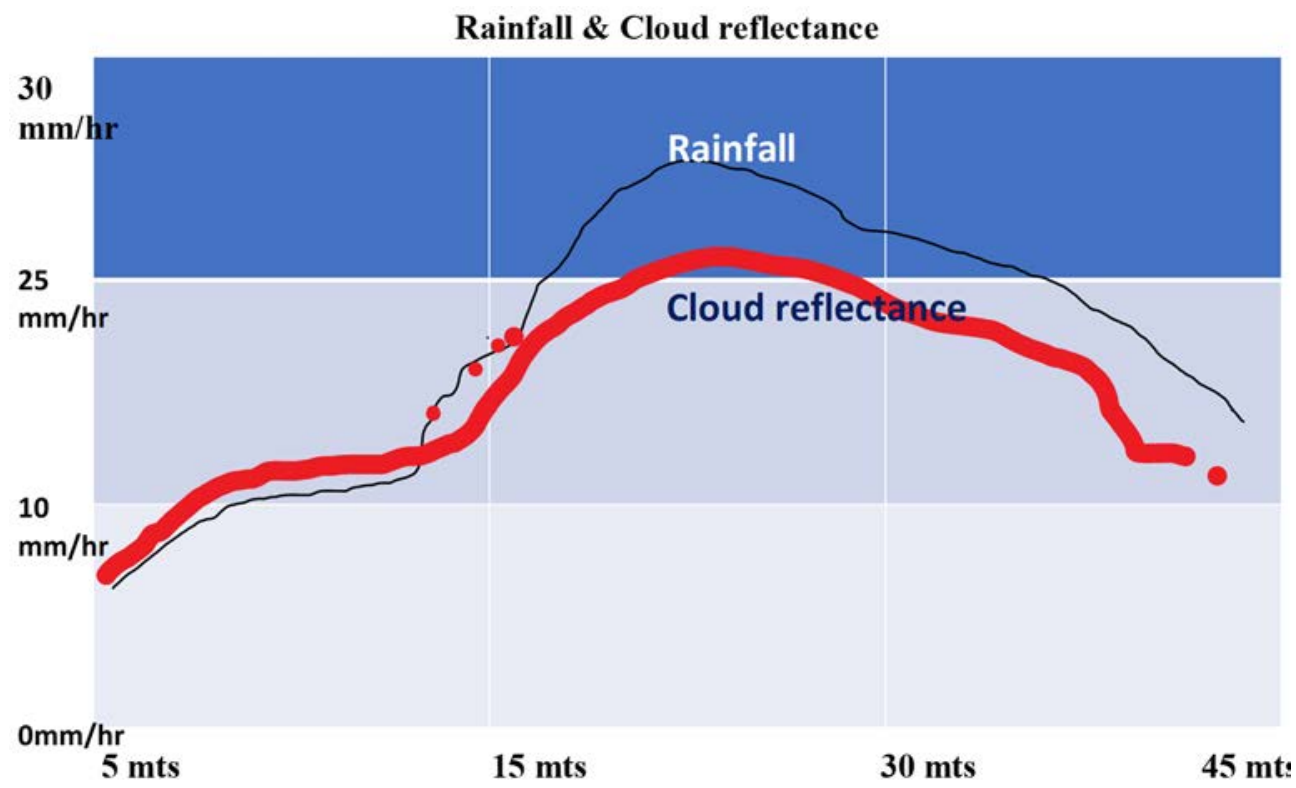

Figure 9: Rainfall and radar reflectance graphs from radar.

\section{CONCLUSIONS}

The cloud seeding operations carried out in Karnataka state in 2017 has been very unique in following aspects:

1. First time in the country two aircrafts operated in the season for cloud seeding there by increasing the target area of operations.

2. Three weather radars were set up to cover the entire state and continuously monitor the weather developments and identify target clouds for seeding.

3. Three Radio Sonde stations data was utilized for generating the daily weather report indicating target districts/taluks/hoblis.

4. Daily weather report using the rainfall data across the state, satellite data and weather model forecasts were prepared meticulously by the Monitoring \& Advisory Team (MAA) indicating specific talukas and hoblis to be given priority for seeding.

5. The operating team and weather experts worked in tandem to maximize the benefit of cloud seeding in the state.

6. The rainfall data from the very vast network of about 6,000 automatic rainguage stations set up by Karnataka State Disaster Management Centre (KSNDMC) helped in identifying priority areas based on antecedent rainfall and also helped to evaluate scientifically the impact of cloud seeding.

\section{REFERENCES}

[1] Anthes, R.A. \& Warner, T.T., Development of hydrodynamic models suitable for air pollution and other meso-meteorological studies. Mon. Wea. Rev., 106, pp. 1045-1078, 1978.

[2] Bigg, E.K., An independent evaluation of a South African hygroscopic cloud seeding experiment, 1991-1995. Atmos. Res., 43, pp. 111-127, 1997. 
[3] Braham, R.R. Jr., Precipitation enhancement: A scientific challenge. Meteor. Monogr., 43, pp. 1-5, 1986.

[4] Bruintjes, R.T., A review of cloud seeding experiments to enhance precipitation and some new prospects. Bull. Amer. Met. Soc., 80, pp. 805-820, 1999.

[5] Bruintjes, R.T. et al., Program for the augmentation of rainfall in Coahuila (PARC): overview and results. Proceedings of 15th Conference on Planned and Inadvertent Weather Modification, Albuquerque, NM, pp. 45-48, 2001.

[6] Koenig, L.R., Numerical modeling of ice deposition. J. Atmos. Sci., 28, pp. 226-237, 1971. 